\title{
Efficacia di selpercatinib nel trattamento dei carcinomi tiroidei con alterazione di RET: risultati di uno studio multicentrico open label, fase $1-2$
}

\author{
Dario Norello ${ }^{1} \cdot$ Alessandro Peri $^{1}$
}

Accettato: 28 gennaio 2021 / Pubblicato online: 11 agosto 2021

(c) The Author(s) 2021

Commento a:

Efficacy of selpercatinib in RET-altered thyroid cancers.

L.J. Wirth, E. Sherman, B. Robinson, B. Solomon, H. Kang, J. Lorch, F. Worden, M. Brose, J. Patel, S. Leboulleux, Y. Godbert, F. Barlesi, J.C. Morris, T.K. Owonikoko, D.S.W. Tan, O. Gautschi, J. Weiss, C. de la Fouchardière, M.E. Burkard, J. Laskin, M.H. Taylor, M. Kroiss, J. Medioni, J.W. Goldman, T.M. Bauer, B. Levy, V.W. Zhu, N. Lakhani, V. Moreno, K. Ebata, M. Nguyen, D. Heirich, E.Y. Zhu, X. Huang,

L. Yang, J. Kherani, S.M. Rothenberg, A. Drilon, V. Subbiah, M.H. Shah, M.E. Cabanillas.

N Engl J Med (2020) 383:825-835

Il proto-oncogene RET codifica per un recettore transmembrana tirosin-chinasico, che può diventare costitutivamente attivato promuovendo l'oncogenesi in seguito a mutazioni o a riarrangiamenti strutturali con fusioni. Mutazioni germinali di RET sono associate a neoplasia endocrina multipla (MEN) 2A e 2B, con una penetranza quasi completa di carcinoma midollare della tiroide. Tali sindromi ereditarie rappresentano il $25 \%$ dei casi di carcinoma midollare tiroideo, mentre nel $60 \%$ dei casi sporadici sono presenti mutazioni somatiche di RET [1].

Nei carcinomi tiroidei non midollari le fusioni di RET sono riscontrabili in meno del $10 \%$ dei casi [2].

Inibitori delle chinasi sono stati approvati per il trattamento del carcinoma midollare della tiroide (vandetanib e cabozantinib) e dei carcinomi differenziati tiroidei refratta-

$凶$ A. Peri

alessandro.peri@unifi.it

1 Dipartimento di Scienze Biomediche Sperimentali e Cliniche "Mario Serio", Università di Firenze, Firenze, Italia ri al radio-iodio (sorafenib e lenvatinib), con percentuali di risposta dal 12 al $65 \%$. La sicurezza e l'efficacia nel tempo di questi farmaci sono limitate dagli effetti tossici dovuti all'inibizione anche di altre chinasi, oltre a RET [3].

Un'importante novità è emersa nel maggio 2020, quando la Food And Drug Administration (FDA) ha autorizzato selpercatinib, inibitore altamente selettivo di RET, per il trattamento del tumore polmonare non a piccole cellule, tumore midollare della tiroide e altri tipi di tumori tiroidei con alterazioni del gene RET.

In particolare, nel trial oggetto di questo articolo, Wirth e collaboratori hanno condotto uno studio open-label multicentrico di fase $1-2$ su pazienti $\geq 12$ anni con carcinoma (avanzato o metastatico) midollare tiroideo con mutazione di RET o carcinoma tiroideo, di qualunque tipo istologico, con fusione di RET. È stato somministrato selpercatinib quotidianamente fino alla progressione di malattia, morte, effetti tossici severi o revoca del consenso.

I risultati hanno evidenziato, in 55 pazienti con carcinoma midollare tiroideo con mutazione di RET precedentemente trattati con vandetanib e/o cabozantinib, una risposta completa o parziale all'imaging, oppure nei valori sierici di calcitonina e CEA, nel 69\% degli individui e una sopravvivenza libera da progressione a un anno dell' $82 \%$.

Invece, in 88 pazienti con carcinoma midollare tiroideo con mutazione di RET, non trattati in precedenza con vandetanib o cabozantinib, è stata riscontrata una risposta completa o parziale nel $73 \%$ dei soggetti e una sopravvivenza libera da progressione a un anno del $92 \%$.

Infine, in 19 pazienti trattati precedentemente con radioiodio e con almeno una terapia sistemica per carcinoma tiroideo (papillare, a cellule di Hürthle, scarsamente differenziato, anaplastico) positivo per fusione di RET, è stata evidenziata una risposta completa o parziale nel $79 \%$ dei casi 
con una sopravvivenza libera da progressione a un anno del $64 \%$.

I principali effetti avversi, di grado $\geq 3$, riportati nello studio sono stati ipertensione (21\%), un incremento dell'alanina aminotransferasi $(11 \%)$ e dell' aspartato aminotransferasi (9\%), iponatriemia (8\%) e diarrea (6\%). Inoltre, nel $2 \%$ dei pazienti il trattamento è stato interrotto per gli effetti avversi correlati al farmaco.

Gli autori concludono il loro lavoro evidenziando come selpercatinib abbia dimostrato nei pazienti trattati un'efficacia duratura con effetti tossici prevalentemente di basso grado.

Il selpercatinib viene quindi proposto come una novità interessante a disposizione del clinico che deve trattare pazienti con tumore midollare della tiroide e altri tipi di tumori tiroidei con alterazioni del gene RET.

Funding Note Open access funding provided by Università degli Studi di Firenze within the CRUI-CARE Agreement.

Nota della casa editrice Springer Nature rimane neutrale in riguardo alle rivendicazioni giurisdizionali nelle mappe pubblicate e nelle affiliazioni istituzionali.
Open Access This article is licensed under a Creative Commons Attribution 4.0 International License, which permits use, sharing, adaptation, distribution and reproduction in any medium or format, as long as you give appropriate credit to the original author(s) and the source, provide a link to the Creative Commons licence, and indicate if changes were made. The images or other third party material in this article are included in the article's Creative Commons licence, unless indicated otherwise in a credit line to the material. If material is not included in the article's Creative Commons licence and your intended use is not permitted by statutory regulation or exceeds the permitted use, you will need to obtain permission directly from the copyright holder. To view a copy of this licence, visit http://creativecommons.org/licenses/by/4.0/.

\section{Bibliografia}

1. Ciampi R, Romei C, Ramone T et al (2019) Genetic landscape of somatic mutations in a large cohort of sporadic medullary thyroid carcinomas studied by next-generation targeted sequencing. iScience 20:324-336

2. Pozdeyev N, Gay LM, Sokol ES et al (2018) Genetic analysis of 779 advanced differentiated and anaplastic thyroid cancers. Clin Cancer Res 24:3059-3068

3. Brose MS, Bible KC, Chow LQ et al (2018) Management of treatment-related toxicities in advanced medullary thyroid cancer. Cancer Treat Rev 66:64-73 